\title{
Electronically driven adsorbate excitation mechanism in femtosecond-pulse laser desorption
}

Brandbyge, Mads; Hedegård, Per; Heinz, T. F.; Misewich, J. A.; Newns, D. M.

Published in:

Physical Review B

Link to article, DOI:

10.1103/PhysRevB.52.6042

Publication date:

1995

Document Version

Publisher's PDF, also known as Version of record

Link back to DTU Orbit

Citation (APA):

Brandbyge, M., Hedegård, P., Heinz, T. F., Misewich, J. A., \& Newns, D. M. (1995). Electronically driven adsorbate excitation mechanism in femtosecond-pulse laser desorption. Physical Review B, 52(8), 6042-6056. https://doi.org/10.1103/PhysRevB.52.6042

\section{General rights}

Copyright and moral rights for the publications made accessible in the public portal are retained by the authors and/or other copyright owners and it is a condition of accessing publications that users recognise and abide by the legal requirements associated with these rights.

- Users may download and print one copy of any publication from the public portal for the purpose of private study or research.

- You may not further distribute the material or use it for any profit-making activity or commercial gain

- You may freely distribute the URL identifying the publication in the public portal 


\title{
Electronically driven adsorbate excitation mechanism in femtosecond-pulse laser desorption
}

\author{
Mads Brandbyge \\ Center for Atomic-scale Materials Physics and Physics Department, Technical University of Denmark, \\ DK-2800 Lyngby, Denmark \\ and Ørsted Laboratory, Niels Bohr Institute, Universitetsparken 5, DK-2100 Copenhagen, Denmark \\ Per Hedegård \\ Ørsted Laboratory, Niels Bohr Institute, Universitetsparken 5, DK-2100 Copenhagen, Denmark
}

T.F. Heinz, J.A. Misewich, and D.M. Newns

IBM Thomas J. Watson Research Center, P.O. Box 218, Yorktown Heights, New York 10598

(Received 22 February 1995)

\begin{abstract}
Femtosecond-pulse laser desorption is a process in which desorption is driven by a subpicosecond temperature pulse of order $5000 \mathrm{~K}$ in the substrate-adsorbate electron system, whose energy is transferred into the adsorbate center-of-mass degrees of freedom by a direct coupling mechanism. We present a systematic theoretical treatment of this coupling process in the language of an electronic friction, which generates Langevin noise in the adsorbate center-of-mass degrees of freedom, while the electronic degrees of freedom are at a high temperature. Starting from an influence-functional path-integral description, a simple formula for the electronic friction is defined which is valid at all electronic temperatures. At low temperatures the formalism makes contact with the electronic friction appearing in the theory of adsorbate vibrational damping, whereas at high temperatures comparable with the adsorbate electronic excitation energies the friction becomes strongly temperature dependent due to dominance by virtual excitations between different adsorbate potential energy surfaces. The former regime is related to the electronic friction model for the desorption process, and the latter to the desorption induced by multiple electronic transistions model for the process; the present formulation comprises both regimes. Desorption is calculated both by a simple quasianalytic Kramers rate approach, and by numerical solution to the Langevin equation. The magnitude of the desorbed fraction and the time scale for desorption are compared to experimental results.
\end{abstract}

\section{INTRODUCTION.}

Femtosecond-pulse laser desorption constitutes a quasithermal reaction process of a nonadiabatic type, ${ }^{1-3}$ as demonstrated in this paper (extending the theoretical picture described in earlier publications). In conventional thermal desorption, the energy transferred into the adsorbate degrees of freedom comes from thermally excited substrate lattice vibrations (phonons). In the laser desorption process discussed in this paper, ${ }^{1-3}$ on the contrary, the energy will be shown to be directly transferred from quasithermally excited electronic states of the substrate-adsorbate complex, a nonadiabatic process that formally violates the Born-Oppenheimer approximation.

The experiments upon which the theory in this paper is based $^{1-3}$ involve a monolayer of molecules, such as NO, $\mathrm{O}_{2}$, or $\mathrm{CO}$, adsorbed on metal surfaces [e.g., $\mathrm{Pd}(111)$ ]. The adsorption energy, e.g., for NO on $\mathrm{Pd}(111)$, is of order $1 \mathrm{eV}$. The laser pulses utilize visible light at $620 \mathrm{~nm}(2$ eV), with duration 100-200 fs. The pulses are widely separated in time, but are relatively intense, giving a desorption probability in the range $10^{-4}-10^{-3}$ per pulse, the desorption probability being a strongly increasing nonlin- ear function of laser fluence. Experimental information and modeling ${ }^{4}$ suggest that the laser photons are mostly adsorbed in the substrate within a skin depth (100 $\AA)$ of the surface, rather than in the adsorbate, eliminating multiphoton adsorption as a significant contribution to the nonlinear cross section.

A consistent picture of the evolution of the surface following absorption of the laser pulse has been reached as a result of modeling and comparison with transient optical reflectivity measurements (see Ref. 1 and references therein). In this picture, quasithermal equilibrium is established in the surface electron gas, which reaches a peak temperature of several thousand $\mathrm{K}$, over a time scale of order several hundred fs (see Fig. 1 below). The relatively small energy in the electron gas (the specific heat of a nearly degenerate electron system is low), is transferred $^{4}$ via electron-phonon coupling to the lattice degrees of freedom, which reach a temperature of only a few hundred $\mathrm{K}$ in a time scale of order $1 \mathrm{ps,} \mathrm{over} \mathrm{which}$ time scale the electron gas cools.

If desorption followed the conventional picture, the energy in the adsorbate would be transferred serially, first from the substrate electronic degrees of freedom into the substrate lattice ("phonon") degrees of freedom, then 


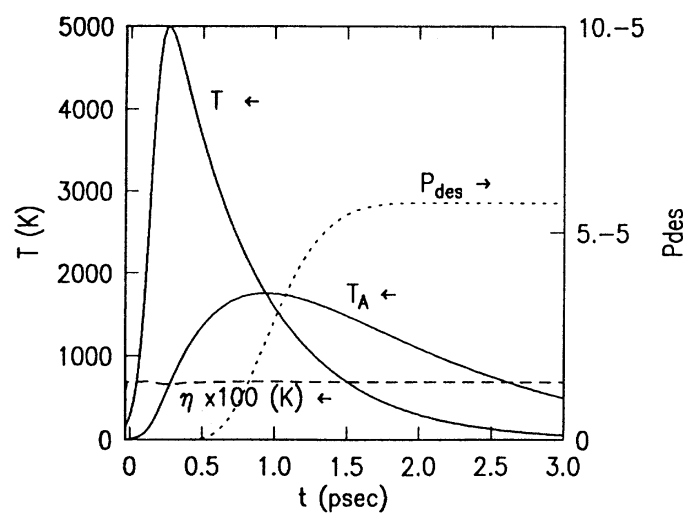

FIG. 1. Time dependence of various quantities in the femtosecond-pulse laser desorption problem, from the solution of Eqs. (19-22), based on a model input for electron temperature $T(t)$. Electronic friction $\eta(t)$, adsorbate temperature $T_{A}(t)$, and the fraction desorbed up to time $t$, are shown. The regime illustrated is nearly constant friction, parameters are $\epsilon_{a}=18000 \mathrm{~K}, C=40000 \mathrm{~K}\left(\epsilon_{\infty}=58000 \mathrm{~K}\right), \Delta^{0}=\epsilon_{a}$, $V_{B}=1.4 \mathrm{eV}, \beta=1.0$ a.u., $\alpha=0.45$ a.u.

into the adsorbate center-of-mass (CM) degree of freedom. We shall see that this scenario is not possible, first on the grounds of available energy, second on the grounds of time scale. Suppose, for example, that the lattice reaches $700 \mathrm{~K}$, and the prefactor in the Arrhenius rate expression is $1 \mathrm{ps}^{-1}$, the adsorption energy is 1 $\mathrm{eV}$, then the desorption probability in $0.4 \mathrm{ps}$ (the actual desorption time scale) is only $3 \times 10^{-8}$, much too low. Moreover the time to reach this lattice temperature is of order $1 \mathrm{ps}$, so $1 \mathrm{ps}$ is a lower limit on the time scale for desorption by the conventional mechanism. However, the desorption time scale ${ }^{3}$ can be determined by measuring the desorption probability in the case of two laser pulses separated by a variable time interval. It is found that the desorption time is remarkably short, about $0.4 \mathrm{ps}$, and is thus incompatible with the conventional mechanism.

The mechanism proposed in this and previous papers is that while electronic energy is being transferred to the lattice degrees of freedom, electronic energy is transferred directly and in parallel to the adsorbate CM and other adsorbate degrees of freedom. We shall demonstrate that this can be relatively efficient, resulting in adsorbate temperatures approaching $2000 \mathrm{~K}$, and on a short time scale, leading to reproduction of the essential experimental features. As mentioned above, in parallel with excitation of the adsorbate degree of freedom, the internal vibrational degree of freedom of the adsorbate is excited. Because of its high frequency $\sim 200 \mathrm{meV}$, this degree of freedom tends to be adiabatically isolated from the lattice degrees of freedom (whose frequencies, being an order of magnitude lower, are ill matched to it), so its excitation acts as a further indication ${ }^{5,6}$ that the underlying excitation mechanism is electronic in nature.

Two initially contrasting frameworks have been employed for thinking about the electronic-vibrational coupling mechanism. One approach is derived from existing knowledge in the field of damping of adsorbate vibrations. These vibrations can excite electron-hole pairs in the metal surface, as pointed out some time ago by Persson and Persson, ${ }^{7}$ providing an electronic channel for the dissipation of energy, which is especially important in the case of high frequency vibrations, which are almost adiabatically isolated from the lattice, ${ }^{7}$ as pointed out above. It is convenient to describe the electronic energy loss process in terms of an electronic friction, ${ }^{8-10}$ acting in the atomic coordinates of the adsorbate. At finite temperature, the friction couples Langevin noise into the adsorbate degrees of freedom. ${ }^{11}$ This idea was earlier proposed as a mechanism for excitation of projectile intramolecular vibration in low-energy scattering of NO from an Ag (111) surface. $^{5}$ In the present problem, we expect there to be also coupling to the adsorbate CM degree of freedom, leading to a desorption mechanism. The concept of an electronic friction driven reaction process was earlier proposed by Bohnen and co-workers ${ }^{8}$ and by Nourtier. ${ }^{8}$

An alternative conceptual framework ${ }^{12}$ has arisen out of the theory of DIET - desorption induced by electronic transitions - at surfaces. The concept here (MGR mechanism) is the excitation of the adsorbate, by a photon or virtual photon, onto an excited, say repulsive, potential energy (PE) surface. The adsorbate then has a tendency to fall down the repulsive curve and to desorb. But due to the close coupling to the surface, the adsorbate may also drop down to the ground state curve with excitation of an electron-hole pair in the metal. Competition by the deexcitation process makes the DIET process very inefficient on metal surfaces, where the deexcitation channel is efficient. However at finite temperatures, there is also a rate for the excitation process from the ground state to the excited state PE curves, equal to the Boltzmann factor times the (large) deexcitation rate. These two rate processes lead to trajectories involving a sequence of excitation and deexcitation processes, which pumps energy into the adsorbate CM degree of freedom, leading to DIMET - desorption induced by multiple electronic transitions. ${ }^{12}$

What is the relationship between these two pictures, electronic friction and DIMET? The electronic friction approach $^{10,11}$ is in the adiabatic picture, being based in its original form on the low excited states of the coupled system consisting of the adsorbate and the surface. On the other hand, the DIMET picture ${ }^{12}$ is based on the diabatic picture, in which the PE curves can be thought of as being defined with the adsorbate-surface coupling turned off, the restoration of the coupling giving rise in this approach merely to the transition rate between the $\mathrm{PE}$ curves. The DIMET picture is able to take into account the highly excited states, for example ionized states, of the adsorbate, but because of the approximate treatment of the adatom-surface coupling, cannot describe the electronic friction. On the other hand, the electronic friction picture in its existing form cannot describe the highly excited electronic, e.g., ionized states, of the adsorbate, and it is clear that these play a significant - even dominant - role at the high electronic temperatures reached in this class of experiment.

In this paper (see also Ref. 13), we give a unified pic- 


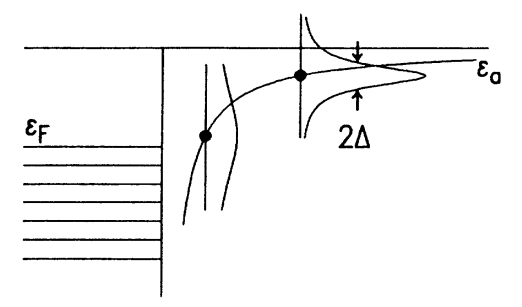

FIG. 2. Behavior of the molecular $\pi^{*}$ affinity level during the approach to the surface. Level shifts down due to the screening by the electron gas - "image force" - [modeled by Eq. (22b)], and broadens [Eq. (22a)] due to tunneling between the molecule and surface. $\epsilon_{a}(z)$ is the affinity level, $\epsilon_{F}$ is the Fermi level, $\Delta(z)$ is the lifetime broadening.

ture of energy transfer from the thermally excited electronic states, both of low energy (frictionlike description) and high energy (DIMET-like description). We do this within a simplified model of the adsorbate and its coupling to the substrate (see Fig. 2). The adsorbate is modeled as having a single electronic level $\epsilon_{a}$, which in the case of the diatomic molecules in question in these experiments presumably represents the $\pi^{*}$ orbital. The level $\epsilon_{a}$ can tunnel into the continuum represented by the substrate conduction band, giving it a lifetime broadening $\Delta$. The electronic ground state of the adsorbate on the surface is represented by the Fermi level intersecting the resonance at $\epsilon_{a}$ at some point.

At adsorbate-surface distances near equilibrium, it seems clear that the characteristic energy scale of the adsorbate electronic structure, represented by the lifetime broadening $\Delta$, is not less than a fraction of an $\mathrm{eV}$, and thus much larger than characteristic inverse time scales for adsorbate motion. This implies that, at least for distances not too far from the surface, the adiabatic picture should be the appropriate one. Therefore in this paper, starting from the adiabatic picture, we derive a version of the electronic friction picture that is valid at all temperatures. The electronic friction is related to the DOS in the adsorbate resonance at the Fermi level, which allows the excitation of low-energy electron-hole pairs. The applicability of the formalism to high temperatures of order $\epsilon_{a}-\epsilon_{F}$ (the separation of the resonance from the Fermi level) means that ionized states of the adsorbate are also implicitly included, essentially including the DIMET physics, even though this is done in the adiabatic picture. We introduce the derivation of our results in the next section, based on a path-integral formalism (most of the details of which are to be found in Appendix A), and give numerical results illustrating the regimes dominated by low-energy excitations (electronic friction) and by high-energy excitations (DIMET) in the following section.

\section{FORMALISM}

Let us now describe the essence of the derivation of the space- and temperature-dependent friction, using the influence-functional method of Feynman and
Vernon. ${ }^{14,15}$ This method is introduced in the Appendix together with a more in-depth treatment of the calculational details outlined in this section.

We consider the problem of an adsorbate with a single valence level at energy $\epsilon_{a}$, interacting with the electron gas at a metal surface. We neglect coupling to phonons. In the language of the influence-functional method, we denote the electronic degrees of freedom as the "reservoir" and the adsorbate as the "system." We assume the electronic part of the problem to be described by a generalized Anderson-Newns type of model, with parameters depending on the adsorbate position $x$,

$$
H_{0}+V_{\text {res-sys }}(x)=\sum_{k} \epsilon_{k} c_{k}^{\dagger} c_{k}+\sum_{k k^{\prime}} V_{k^{\prime} k}(x) c_{k}^{\dagger} c_{k^{\prime}} .
$$

Here, $k$ denotes all the electronic states including the adsorbate state, $k=a$, and

$$
V_{k k^{\prime}}(x)=T_{k}(x) \delta_{k^{\prime} a}+T_{k^{\prime}}^{*}(x) \delta_{k a},
$$

so the hybridization terms as well as the adsorbate energy,

$$
\varepsilon_{a}(x)=\epsilon_{a}+2 \operatorname{Re}\left[T_{a}(x)\right],
$$

depend on $x$. The spin is ignored. This somewhat compact notation will be very convenient in the calculations that follows. Besides this part of the adsorbate Hamiltonian, the adsorbate is assumed to move in some potential confining it to the surface. In the case of no occupation of the valence level, the adsorbate will be moving on this "ground state" potential energy surface (PES), $V_{g}(x)$, while the transfer of an electron to the valence state will cause the adsorbate to move on the "excited state" PES, $V_{e}(x)$

$$
V_{e}(x)=V_{g}(x)+\varepsilon_{a}(x) .
$$

As in Ref. 12, here we only take two PES's into account. The transition rate between $V_{e}$ and $V_{g}$ is governed by the matrix elements $T_{k}(x)$.

With this Hamiltonian as our starting point, we will now derive the Langevin-equation description of the motion of the adsorbate. Assume that the electronic system is in thermal equilibrium at temperature $1 / \beta$ at a certain time $t=0$. We can then write the total density operator as,

$$
\rho(0)=\rho_{\text {sys }}(0) \otimes \frac{1}{Z} e^{-\beta H_{0}} .
$$

All relevant information about the adsorbate motion is contained in the so called reduced density operator of the system. The density operator evolves in time according to $\rho(t)=e^{-i H t} \rho(0) e^{i H t}$. We will represent the system part of the time-evolution operators as path integrals in the standard way, first described by Feynman:

$$
e^{-i H t}=\int \mathcal{D} x e^{i S_{0}(x)} U(x, t) .
$$

Here, $U(x, t)$ is the time-evolution operator of the environment (the electron system in our case) in the presence 
of a time dependent "field" $x\left(t^{\prime}\right)$ - given by a particular path of the adsorbate. We get one such factor from both $e^{-i H t}$ and $e^{i H t}$, so that the final reduced density operator, which is the total density operator traced over all electron states, can be written as a double path integral:

$$
\rho_{\mathrm{red}}(t)=\int \mathcal{D} x \int \mathcal{D} y e^{i S_{\mathrm{o}}(x)-i S_{\mathrm{o}}(y)} \mathcal{F}_{\mathrm{el}}(x, y) \rho_{\mathrm{sys}}(0)
$$

where $S_{0}$ is the action for the adsorbate decoupled from the electronic environment. $\mathcal{F}_{\mathrm{el}}(x, y)$ is the influence functional. It contains the effect of the electronic degrees of freedom on the adsorbate motion. We can write it in terms of the electronic Hamiltonian and define the effective action describing the adsorbate motion,

$$
\begin{aligned}
\mathcal{F}_{\mathrm{el}}(x, y)= & \frac{1}{Z} \operatorname{Tr}_{\mathrm{el}}\left[e^{-\beta H_{0}} T e^{i \int_{0}^{t} d t^{\prime}\left[H_{0}+V\left(y\left(t^{\prime}\right)\right)\right]}\right. \\
& \left.\times \hat{T} e^{-i \int_{0}^{t} d t^{\prime}\left[H_{0}+V\left(x\left(t^{\prime}\right)\right)\right]}\right] \\
= & \left\langle U(y, t)^{\dagger} U(x, t)\right\rangle_{\mathrm{el}} \equiv e^{i \Delta S_{\mathrm{eff}}(x, y)}
\end{aligned}
$$

Here \langle\rangle$_{\mathrm{el}}$ is the thermal average over the electronic states. $T$ and $\hat{T}$ are time-ordering operators. The evolution $U(y, t)^{\dagger} U(x, t)$ can be viewed as an evolution along the contour, $\gamma$, shown in Fig. 3 below, and $\Delta S_{\text {eff }}$ can be calculated in a simple way using the linked cluster expansion for this contour evolution. To carry out the calculation, the contour Green's functions is mapped onto the ordinary real-time Green's functions by the standard Keldysh method.

We can think of the important paths in the path integral as the classical path plus small fluctuations. The classical path is associated with the "diagonal" parts of the density matrix $(x=y)$, and the fluctuations is associated with the deviations from the classical path, where $x \neq y$. To see this, neglect for a moment the influence functional, i.e., set $\mathcal{F}_{\text {el }}=1$. Write the two paths $x(t)$ and $y(t)$ as an average path $Q(t)=[x(t)+y(t)] / 2$ and a difference path $\xi(t)=x(t)-y(t)$. Expand the actions to second order in $\xi$ to get

$$
S_{0}(x)-S_{0}(y)=-\int_{0}^{t} d t^{\prime}\left[m \ddot{Q}\left(t^{\prime}\right)+V^{\prime}\left(Q\left(t^{\prime}\right)\right)\right] \xi\left(t^{\prime}\right)
$$

If this was the complete action, the functional integral over $\xi$ would result in a (functional) $\delta$ function, which would ensure that the average path $Q$ satisfy Newton's equation,

$$
m \ddot{Q}+V^{\prime}(Q)=0
$$

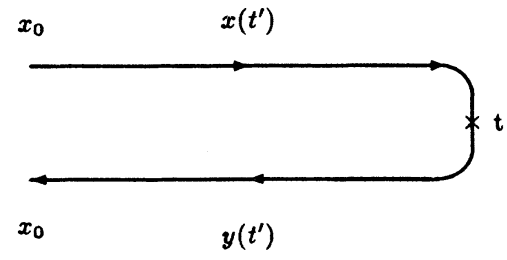

FIG. 3. The contour $\gamma$.
For the harmonic oscillator, this would be exact. This result is well known. The density matrix for a harmonic oscillator results in a Wigner phase-space distribution function, which is identical to the classical phase-space distribution function. Quantum effects only come in through the initial states, which in the quantum case of pure states must obey the uncertainty principle. For potentials that are not harmonic, quantum effects enter also through higher order terms in $\xi$. The effect of the environment is, as we shall see, the introduction of 1 and 2 order terms in $\xi(t)$, thereby converting the functional $\delta$ function into a Gaussian with a width, characteristic of environment.

In general, $\Delta S_{\text {eff }}$ contains both a real and an imaginary part. It is the imaginary part that tends to reduce quantum coherence by exponentially damping paths, where $x$ and $y$ deviate on the classical time scales. In the "semiclassical" picture, we can relate the imaginary part to the fluctuating forces acting on the adsorbate, due to the coupling to the electronic environment, as emphasized by Caldeira and Legget. ${ }^{16}$ The real part contains an exact time-local contribution, which precisely equals the change in free energy, $F(x)$, for the adsorbate at the given position, $x$, resulting in a temperature-dependent force. Besides this time-local part, the real part contains $\dot{x}$ terms related to the dissipation of energy to the electronic system, i.e., friction.

The characteristic electronic time scale is set by the lifetime of the adsorbate state, the inverse of its lifetime broadening,

$$
\Delta(\epsilon, x)=\pi \sum_{k}\left|T_{k}(x)\right|^{2} \delta\left(\epsilon-\epsilon_{k}\right)
$$

while the strength of the coupling between the adsorbate and the electronic environment is controlled by the time derivatives of the adsorbate-state broadening, $\Delta(x)$, and energy, $\epsilon_{a}(x)$, with respect to $x$. In the limit of short lifetime/large broadening the picture of motion on the $V_{g}$ PES with transitions to the $V_{e}$ PES is not feasible, because these transitions occur on a time scale many times faster than the motion of the adsorbate. In this limit, it is more reasonable to consider the motion on the PES given by the effective potential $V_{\text {eff }}=V_{g}(x)+F(x)$ combined with dissipation of energy to the electronic system, expressed by the $\dot{x}$ terms in the effective action. In this limit, it could be reasonable to employ a masterequation approach as in Ref. 11, using the eigenstates of $p^{2} / 2 M+V_{\text {eff }}(x)$ as the basis, but these will only be well defined in the case of low friction. In the other limit, where the adsorbate state is longlived, the DIMET picture with two PES used in Ref. 12 is more appropriate.

Now, our goal is, on the basis of the effective action, to justify the use of the Langevin-equation approach, and especially from Eq. (8) to find the appropriate electronic friction coefficient, $\eta$, which in this case depend on the position of the adsorbate and temperature of the electronic environment. To achieve this, we follow Schmid, ${ }^{17}$ who has shown how a "semiclassical" Langevin equation can emerge from a certain Gaussian influence functional in the case of a particle moving in a general potential. The 
"semiclassical" Langevin equation is justified for a linear system-environment coupling, in the case where quantum fluctuations, due to terms beyond the harmonic in the potential are negligible. The electronic friction picture where $\eta$ is well defined will only make sense in the limit where the electronic time scale is much faster than the time scale of adsorbate motion $\left(\omega_{0} \ll \Delta\right)$, so the electronic environment acts as noise on the adsorbate motion.

To calculate the effect of the fluctuations, we use the following setup. First, we assume that the "forward" and "backward" moving paths, $x$ and $y$, in (8) only deviate from each other in time intervals that are short compared to typical times in semiclassical motion, and in amounts that warrant an expansion of the effective action to second order in the difference $\xi=x-y$. We find, using realistic parameters, that the duration of these deviating paths is indeed very small compared to the "semiclassical" time scales in agreement with our starting assumption and we have obtained a self-consistency.

The friction term entering the Langevin equation will be of second order in $V_{k k^{\prime}}^{\prime}(Q)$. When the lifetime broadening of the adsorbate is on an energy scale of eV's and the adsorbate path moves with frequencies on the scale of meV's, we can argue that the friction is local in time. Including this lowest order friction term and the infinite order free energy in the effective action, we get the reduced density matrix,

$$
\int \mathcal{D} Q \int \mathcal{D} \xi e^{\frac{i}{\hbar} \int_{0}^{t} d \tau\left[M \ddot{Q}(\tau)+V_{\mathrm{eff}}^{\prime}(Q)+\eta(Q) \dot{Q}(\tau)\right] \xi(\tau)-\frac{1}{\hbar} \int_{0}^{\delta} d \tau \int_{0}^{\delta} d \sigma \xi(\tau) K(\tau-\sigma) \xi(\sigma)}
$$

where $(Q, \xi)$ are the rotated variables, $[(x+y) / 2, x-y]$, and $V_{\text {eff }}$ has been expanded around $x=y$. A probabilistic interpretation of this path integral is equivalent to the Langevin equation ${ }^{17}$ describing the adsorbate motion,

$$
\begin{aligned}
& M \ddot{Q}+V_{\text {eff }}^{\prime}(Q)+\eta(Q) \dot{Q}=f(t), \\
& \langle f(t) f(0)\rangle=K(t) .
\end{aligned}
$$

We find that $K$ obeys the fluctuation-dissipation theorem. We postpone all mathematical details concerning this derivation to the Appendix.

Our calculation results in the following expression for the temperature- and space-dependent friction, written in terms of the instantaneous phase shift, $\delta(\epsilon, x)$ (adsorbate mass taken as unity):

$$
\eta(Q)=-\int_{-\infty}^{\infty} d \epsilon \frac{1}{\pi}\left(\frac{\partial \delta}{\partial x}(\epsilon, Q)\right)^{2} \frac{d n_{F}}{d \epsilon}(\epsilon)
$$

where we see that temperature dependence is important only for temperatures on the order of the electronic energy scale defined by the quantities $\Delta$ and $\epsilon_{a}-\epsilon_{F}$. In the limit of zero temperature, we get

$$
\eta(x)=\frac{1}{\pi}\left(\frac{\partial \delta}{\partial x}\left(\epsilon_{F}, x\right)\right)^{2},
$$

which is the result used in Ref. 11. The free energy can also be written in terms of this phase shift,

$$
F(x)=-\frac{1}{\pi} \int_{-\infty}^{\infty} d \epsilon n_{F}(\epsilon) \delta(\epsilon, x) .
$$

Equation (17) leads to a thermal shift in the ground state PE surface, which, however, turns out to be small in the practical range of parameters, and we shall neglect it in the following.

\section{ANALYTIC APPROACH}

In this section, we present some essentially analytic results obtained for the simple model of a truncated har- monic well and a spatially independent friction. The Langevin equation with a local friction can more conveniently be written in terms of a Fokker-Planck equation for the purpose of an analytic solution. We consider a probability distribution $W(\epsilon, t)$ for the adsorbate to have energy $\epsilon$ above the bottom of the well (of depth $V_{B}$ ), which obeys, in the classical limit, the Fokker-Planck equation,

$$
\frac{\partial W}{\partial t}=\eta(t) \frac{\partial}{\partial \epsilon}\left(\epsilon\left[1+T(t) \frac{\partial}{\partial \epsilon}\right] W\right)
$$

equivalent for a harmonic well to a Langevin equation with friction $\eta$ and reservoir temperature $T$.

For the desorption problem, a solution is required with the boundary conditions (a) at $t=0 W(\epsilon, t)=\delta(\epsilon)$ (distribution initially concentrated at bottom of well), and (b) $W\left(V_{B}, t\right)=0$ at all times, corresponding to the Kramers low-friction limit ${ }^{18}$ (electronic friction is normally small).

The solution is obtained in terms of an auxiliary function, the "adsorbate temperature," $T_{A}(t)$, which obeys the equation

$$
\frac{d T_{A}}{d t}+\eta(t) T_{A}=\eta(t) T(t) .
$$

In Ref. 11 we showed that, by introducing an "image" solution with negative sign in the region $\epsilon>V_{B}$, it was possible to satisfy the boundary condition (b) (for details we refer the reader to Ref. 11). In the regime appropriate for the present problem, in which temperatures are small compared with the well depth, the desorption probability obtained is

$$
P_{\mathrm{des}}=V_{B} \int_{0}^{\infty} d t \frac{\eta(t)}{T_{A}(t)} e^{-V_{B} / T_{A}(t)}
$$

Note that this result is just the time integral over the Kramers low-friction rate $R_{K}$, which, in the case of constant temperature, would be $R_{K}=$ $\left(V_{B} \eta / T_{A}\right) \exp \left(-V_{B} / T_{A}\right)$. In the pulsed laser desorption 
problem, the temperature entering the Kramers rate is the adsorbate temperature $T_{A}$, not the electronic temperature $T$. In some sense, a "two step" picture of the desorption process emerges: that energy has to be transferred from the electronic degrees of freedom at temperature $T$ via (19) into the adsorbate degree of freedom to reach temperature $T_{A}$, desorption then occurs as a result of this temperature via (20). Both the rise in adsorbate temperature and the desorption process are both controlled by the same friction parameter $\eta$.

We may use this crude model to get an estimate of the desorption behavior. We include a spin degeneracy of 2, together with an orbital degeneracy of 2, and make explicit the mass $M$ of the adsorbate center of mass, to obtain

$$
\eta=-\frac{4 \hbar}{\pi M} \int_{-\infty}^{\infty} d \epsilon\left(\frac{\partial \delta(\epsilon, x)}{\partial x}\right)^{2} \frac{d n_{F}(\epsilon)}{d \epsilon}
$$

The phase shift is taken to be of the form $\mathrm{A}(54)$, except that in the usual wide band approximation, the shift $\mathcal{H}$ is assumed constant and is absorbed into $\epsilon_{a}$.

The model for the spatial dependences of $\Delta$ and $\epsilon_{a}$ is (see Fig. 2)

$$
\begin{gathered}
\Delta=\Delta_{0} e^{-\beta x}, \\
\epsilon_{a}=\epsilon_{\infty}-C e^{-\alpha x} .
\end{gathered}
$$

Since the quasianalytic treatment can handle time-but not spatially - varying friction, we assume that the equilibrium distance is the "typical" distance, calculating $\eta(t)$ from (21) and (22) at that point, and inserting the result into (19-20).

Results are presented in Figs. 1 and 4 for two cases. Figure 1 displays a case where the resonance is broad and near the Fermi level, with DOS at the Fermi level being relatively large, so that the friction is large at zero temperature and relatively temperature independent. In Fig. 1, we see that the adsorbate temperature rises significantly, peaking at $2000 \mathrm{~K}$, and there is significant desorption. In Fig. 4, a case is illustrated where the resonance is narrow and relatively far above the Fermi level so that the low temperature friction is relatively small.

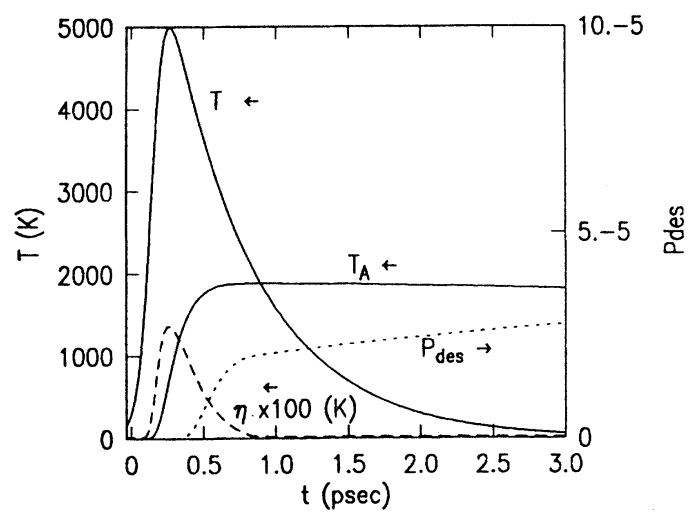

FIG. 4. Time dependence of various quantities in the femtosecond-pulse laser desorption problem, DIMET case, parameters as in the previous figure, except for $\Delta^{0}=0.08 \epsilon_{a}$.

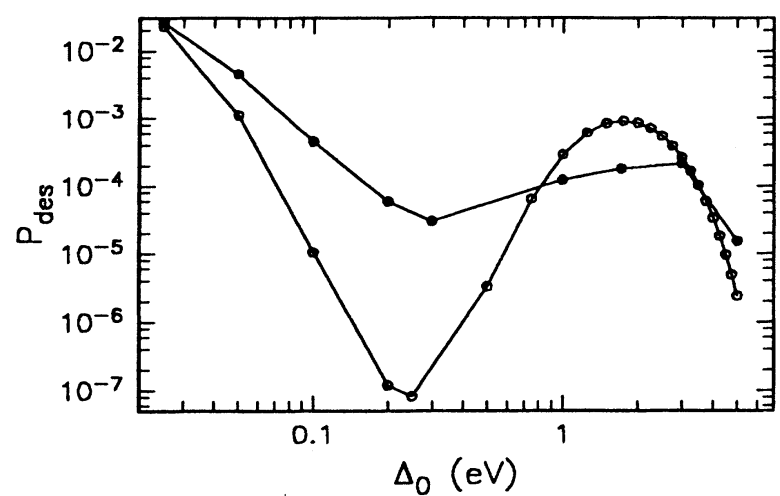

FIG. 5. Plot of desorption probability as a function of $\Delta^{0}$, parameters are $C=3.02 \mathrm{eV}, \epsilon_{\infty}=5 \mathrm{eV}, V_{B}=1.4 \mathrm{eV}, \beta=$ 1.0 a.u., $\alpha=0.5$ a.u. Open circles, analytic calculation, full circles, and simulation are described in the next section.

The excitation energy $\epsilon_{a}-\epsilon_{F}$ has been chosen to be accessible by the given electronic temperature (DIMET-like process), so there is now a rapid rise in the friction as the electronic temperature rises. Again with the parameters in Fig. 4, there is a substantial rise in adsorbate temperature, and significant desorption.

Figure 5 presents results as a function of $\Delta^{0}$, covering the whole range between the friction and DIMET regimes. It is seen that, in fact, these form welldemarcated regimes, the DIMET regime to the left of the figure, and the friction regime to the right, there being a lower desorption probability in between.

A clear experimental distinction between models where friction is primarily the low temperature one, and where it is the DIMET-like thermally induced friction, is seen from the perspective of the time scales. A measure of the time scale involved is available in the measurements of desorption probability in experiments with two equal laser pulses separated by time $\tau$, in which desorption probability is measured as a function of $\tau$. The two-pulse experiment is modeled in Fig. 6, where it is seen that the

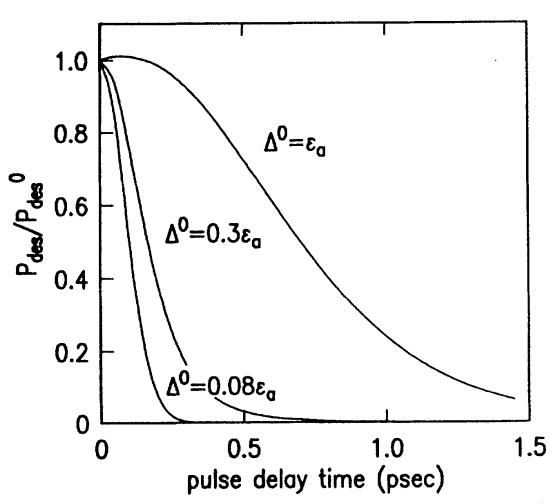

FIG. 6. Two-pulse experiment, in which a laser pulse corresponding to the parameters of Figs. 1 and 4 is split into two equal components separated by a pulse delay time. The desorption probability is plotted as a function of delay for three values of $\Delta^{0}$, other parameters are as in Figs. 1 and 4. 
DIMET type of parameter regime leads to a faster desorption time (as measured in the two-pulse experiment) than the low temperature friction type of regime. This is because desorption in the DIMET regime can only occur in the brief time span when both friction and adsorbate temperature are large, while in the case of temperatureindependent friction, the less retrictive condition that the adsorbate temperature be large is sufficient, satisfiable in a less stringent time frame. The experiments require a rather short "desorption time" of order $0.4 \mathrm{ps,}$, and are thus more compatible with the DIMET-type regime, or a regime with at least partially activated friction.

\section{NUMERICAL RESULTS}

In this section, we present results from a numerical solution to the Langevin equation. This allows us to move beyond the truncated harmonic potential well approximation used in the analytic approach of the previous section with the introduction of more realistic potential energy surfaces. In addition, the numerical approach allows the use of a friction varying with position in the potential as suggested by Eqs. (21) and (22) in the previous section. These results will allow us to test the applicability of the analytic approximations of the previous sections.

The well-known Langevin equation describes the trajectory of the center-of-mass coordinate of the adsorbate, $x$ :

$$
\frac{d^{2} x}{d t^{2}}+\eta(x, t) \frac{d x}{d t}=\frac{F(x)}{M}+\Gamma(t)
$$

where $M$ is the adsorbate mass and $\eta(x, t)$ is the friction calculated from Eqs. (21) and (22) for an adsorbate at center-of-mass position $x$. The friction depends on the time through the temporal dependence of the substrate electronic temperature, $T(t)$. Here $F(x)=-d V(x) / d x$ is the force exerted on the molecule as a result of the potential energy surface, $V(x)$, and $\Gamma(t)$ is a stochastic force with mean $\langle\Gamma(t)\rangle=0$. We take $\Gamma(t)$ to be described by a white noise spectrum:

$$
\left\langle\Gamma(t) \Gamma\left(t^{\prime}\right)\right\rangle=q \delta\left(t-t^{\prime}\right),
$$

where the strength of the white noise term, $q$, is determined by the temperature, $T$, and friction, $\eta$, through a fluctuation-dissipation theorem:

$$
q=\frac{2 \eta k T}{M} \text {. }
$$

This formalism, in conjunction with the expression for the friction described above, is examined through stochastic trajectory simulations using standard methods for integration of the Langevin equation.

The adsorption well is modeled using a Morse potential. The depth of the well and the curvature at the equilibrium position were chosen to match the well depth $(1.37 \mathrm{eV})$ and molecule-surface vibration quantum (0.032 $\mathrm{eV}$ ) for the NO/Pd(111) system, which was used in femtosecond laser desorption experiments. ${ }^{1}$ This potential is

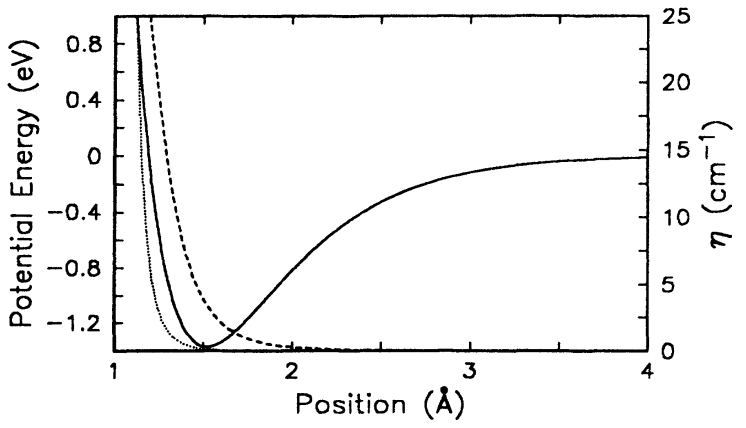

FIG. 7. Morse potential used in numerical integration of Langevin equation (solid line). Also shown is the friction as a function of position in the well at $400 \mathrm{~K}$ (dotted line) and $5500 \mathrm{~K}$ (dashed line).

shown in Fig. 7. The temperature transient used in the simulations comes from a model for the electronic temperature of the substrate under ultrafast laser excitation conditions, which was used to describe the femtosecond laser desorption experiments. ${ }^{1}$ This temperature transient starts at $400 \mathrm{~K}$ and rises to $5500 \mathrm{~K}$ over the width of the laser pulse (100 fs) and then falls approximately exponentially with a time constant of about 1 ps to the equilibrium value. For all the calculations presented here, the parameters used to calculate the friction are $\alpha=0.5$ a.u., $\beta=1$ a.u., $\epsilon_{\infty}=5 \mathrm{eV}$, and $\mathrm{C}=3.02 \mathrm{eV}$. Using a width, $\Delta_{0}=0.2 \mathrm{eV}$, we also show in Fig. 7 the spatial variation of the friction at two temperatures, $400 \mathrm{~K}$ and $5500 \mathrm{~K}$. The dependence of the friction on temperature at well minimum is shown in Fig. 8.

The results of a series of stochastic trajectory simulations are shown along with the analytic results of the previous section in Fig. 5, where the desorption probability is plotted for several values of the width of the affinity level at the well minimum, $\Delta_{0}$. The results show similar qualitative trends with $\Delta_{0}$. At large $\Delta_{0}$, the friction becomes temperature independent and the desorption probability maximizes at a relatively low value. As the width $\Delta_{0}$ decreases, the desorption probability decreases as the friction decreases. At lower values of $\Delta_{0}$, however, the physical picture transforms to one more sim-

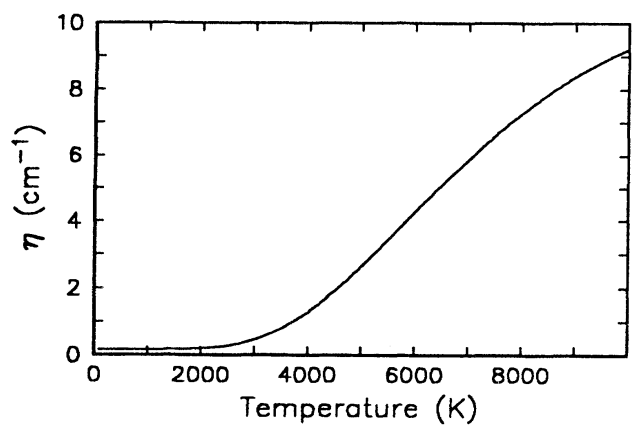

FIG. 8. Friction as a function of electronic temperature calculated at the well minimum. 
ilar to the DIMET picture. ${ }^{12}$ The system now becomes that of a well-defined affinity level lying above the Fermi energy. Although the low temperature friction continues to decrease as $\Delta_{0}$ decreases, the temperature dependence of the friction becomes much more pronounced. In this regime, the increased high temperature friction leads to an increase in desorption probability as the width $\Delta_{0}$ decreases. As seen in Fig. 5, a significant increase in the desorption probability can result in the "DIMET" regime.

\section{CONCLUSION}

In our description of femtosecond-pulse laser desorption, the laser energy is converted into a transient temperature pulse in the electron gas at the substrate surface. The pulse is very high $(\sim 5000 \mathrm{~K})$, but very short $(\sim 200$ fs.). Due to the shortness, plus the low heat capacity of the electron gas, the lattice temperature eventually reached is low (and the lattice undamaged). Transmission of energy from the electron gas into the adsorbate nuclear degrees of freedom occurs via the dependence of the molecule's electronic properties on the ionic positions. Additionally knowledge of the molecule's potential energy surface is required.

We have implemented a modelistic version of these assumptions. Only the molecular center-of-mass degree of freedom is treated in the desorption problem, and only motion in one dimension, involving a Morse-type potential well for the molecule, is considered. The adsorbate electronic structure is defined by an Anderson-Newns type of model in terms of a resonance, whose width $\Delta(z)$ and center $\epsilon_{a}(z)$ vary with distance from the surface. The resonance can be thought of as the $\pi^{\star}$ orbital, usually believed to dominate the low-energy electronic phenomena in first row diatomic adsorbates, such as $\mathrm{NO}$ and $\mathrm{O}_{2}$.

The theory of an object, whose electronic structure depends on position, coupled to a translationally heated electron gas can be formulated generally using the influence-functional theory of Feynman and Vernon. To make further progress, it is assumed that in this problem, the electronic time scale $\Delta^{-1}$ is shorter than the ionic motion time scale, which should be valid in the neighborhood of the equilibrium position, though it will eventually break down at sufficiently large distances, due to the exponential vanishing of $\Delta$.

When the relative shortness of the electronic time scale holds, it is carefully demonstrated in Appendix A that the electron gas-to-adsorbate energy transfer process is describable in terms of an electronic friction $\eta$. The electronic friction couples Langevin noise from the electronic heat bath into the adsorbate center-of-mass degree of freedom in the conventional way, resulting in a Brownian motion which may result in desorption. When it arises from direct energy (rather than momentum) transfer to the substrate electron gas, electronic friction can be rather simply thought of as follows. ${ }^{13}$ At each position there will be a given amount of charge transfer into the adsorbate $\pi^{\star}$ resonance. Adsorbate motion leads to a current $I$ into the resonance, and hence to a power ab- sorption $I^{2} / G$, which can be straightforwardly related to electronic friction. When this is done, it is recognized that $G$ is none other than two units of the quantum of conductance $G^{0}=e^{2} / h$. The quantization originates in the constraint involved in postulating that only a single orbital (the $\pi^{\star}$ ) is involved in charge transfer. The friction is, in general, $T_{e}$ dependent, due to the possibility of thermally aided electron transfer into an adsorbate excited electronic state.

We adopted two approaches to solving the problem in which the adsorbate center of mass is coupled by electronic friction to the transiently heated electron gas bath. An analytic approach, specialized to the model of a truncated harmonic oscillator potential well and spatially invariant friction, yields desorption probability as the time integral over the Kramers low-friction desorption rate. In the expression for the Kramers rate, the temperature that enters is the "adsorbate temperature," a quantity which obeys a simple equation analogous to Newton's law of cooling for the adsorbate, with the electron gas as the heat bath. A numerical approach, involving running a set of trajectories for the Langevin equation, enables an arbitrary well shape and a spatially varying friction to be handled.

The results of the two approaches, which broadly agree, show that parameters fall into two quite welldemarcated regimes separated by a region of low desorption probability. If the width $\Delta$ of the adsorbate resonance is narrow relative to its energy measured from $\epsilon_{F}$, then the dominant contribution to the friction is due to thermal excitation from the Fermi energy to the resonance. This leads to a highly $T$-dependent friction. Since by hypothesis the resonance is narrow compared with its excitation energy, the result can be interpreted in terms of repeated excitation between ground state and excited (negative ion) state PE curves. This mechanism for desorption in the femtosecond-pulse laser context was earlier introduced on a more empirical basis as DIMET (Ref. 12). An opposite regime occurs when the resonance width $\Delta$ is large compared with $T$ and of the order of the distance of the resonance from the Fermi level. Now low-energy electron-hole pairs dominate the friction $^{11}$ channel, and in the regime we are describing, they give rise to a relatively $T$-independent friction.

In the present paper, we have brought the DIMET and $T$-independent friction regimes into the same formal framework (previously the former was done only numerically and the latter analytically), enabling them to be compared. This is done in Fig. 4. The two approaches essentially agree. The shallower dip given by the numerical approach between the two regimes is attributable to the spatially varying friction in the numerical model, which can find a spatial location where the parameters are optimal for the friction, under conditions when the more rigid constraints of the analytic model confine it to a very unfavorable parameter space.

Experimental data can provide some criteria for determination of the parameter regime in experimental systems such as $\mathrm{NO} / \mathrm{Pd}(111)$. We will do this primarily on the basis of the calculations presented in Fig. 5. First the definition of the low- $T$ friction regime as lying to the 
right of the minimum implies a low- $T$ friction of at least $0.2 \mathrm{~cm}^{-1}$. In fact, the work of Ref. 23 suggests that values as large as 1 to a few $\mathrm{cm}^{-1}$ are reasonable (which are required at the maximum desorption probability for this regime). An extremely low low- $T$ friction would, however, point to the DIMET regime. The resonance width at the equilibrium position in the DIMET regime in Fig. 5 should lie below $0.25 \mathrm{eV}$. This value is on the low side for available data such as inverse photoemission. ${ }^{24}$ A third test is provided by measurements of the desorption probability in a two-pulse experiment. ${ }^{3}$ Modeling this quantity as a function of the time separating the pulses (see Fig. 6) reveals that this experiment distinguishes the low- $T$ friction and DIMET regimes in giving a shorter time scale to the latter process. This is because the friction in DIMET only remains high during the short period when the electron temperature is near its peak. Since experiments ${ }^{3}$ favor a rather short characteristic time of $0.4 \mathrm{ps}$, this indicates that a degree of activation of the friction is occurring, so that the DIMET regime or the low- $\Delta$ part of the friction regime is probably operative.

There are some other experimental signatures that we have omitted from the relatively narrow focus of this paper. First, the strong vibrational excitation of the adsorbate is most likely occurs via the electron-hole mechanism discussed in an earlier work on the vibrational excitation observed in NO scattered from $\mathrm{Ag}(111),{ }^{5}$ and is characteristic of desorption at high electron temperature. In the present case, a generalization to include the temperature dependence of the friction is appropriate. A discussion has been given in the literature by two of us. ${ }^{12,6}$ The kinetic energy of the desorbed particles is also of interest. Numerical simulations show it to be relatively low (below $1000 \mathrm{~K}$ ), which is in agreement with the experimental data.

Some of the approximations made in the present paper have been used by other authors. Head-Gordon and Tully ${ }^{10}$ have considered additional degrees of freedom of the adsorbate, but in the context of a finite cluster description in which the electronic states are discrete instead of forming a continuum, with some penalty in the accuracy of the description of the low-energy excitations and thus the friction. The role of substrate phonons has been neglected in the present paper; they act to reduce desorption by trying to cool the adsorbate to their own low temperature. Their effect has been discussed by Billing. ${ }^{25}$

In conclusion, the role of electronic friction in at least one elemental surface reaction process seems to be fairly well established in the case of femtosecond-pulse laser desorption. It may be that electronic friction has a significant effect in other reaction processes, where experimental conditions do not permit its role to be so cleanly separated as in the present situation.

\section{APPENDIX A: TEMPERATURE-DEPENDENT FRICTION}

Here, the influence functional by Feynman and Vernon ${ }^{14,15}$ is introduced. Later, we will discuss its con- nection to the "semiclassical" Langevin equation, following Caldeira and Legget ${ }^{16}$ and Schmid, ${ }^{17}$ and calculate the appropriate (temperature-dependent) friction coefficient to be used in the Langevin equation.

In general, we consider a quantum system with few degrees of freedom, interacting with a general reservoir, i.e., a continuum of degrees of freedom, and we are interested in the influence of the reservoir on this system. All information about the system plus reservoir is contained in the total density operator ( $\hbar \equiv 1$ this appendix),

$$
\rho(t)=e^{-i H t} \rho(0) e^{i H t} .
$$

For the system we will use the coordinate representation, while the representation for environment will remain unspecified. As discussed in the main text, we will represent the evolution of the system as path integrals. In Ref. 19, it is shown how to do this in detail. The result for a single evolution operator $e^{-i H t}$ is

$$
\left\langle x_{1}\left|e^{-i H t}\right| x_{2}\right\rangle=\int \mathcal{D} x e^{i S_{0}(x)} U(x, t) .
$$

Here, $S_{0}(x)$ is the classical action of the uncoupled particle for the path $x(t)$ moving from $x_{1}$ to $x_{2}$, and $U(x, t)$ is the reservoir time evolution operator with a time-dependent Hamiltonian corresponding to a situation, where the particle moves along a $c$-number path $x(t)$ :

$$
U(x, t)=T e^{-i \int_{0}^{t} d t^{\prime} H_{\mathrm{ress}}+H_{\mathrm{int}}\left(x\left(t^{\prime}\right)\right)} .
$$

The reduced density matrix is defined by

$$
\rho_{\text {red }}(t)=\operatorname{Tr}_{\text {res }}[\rho(t)]
$$

where the trace is over a complete set of states for the reservoir. With the above, it is now straightforward to write down a path-integral expression for the reduced density matrix. It will be an integral over pairs of paths $x$ and $y$, since the density matrix involves two time evolution operators $e^{-i H t}$ and $e^{i H t}$ :

$$
\begin{aligned}
\left\langle x_{1}\left|\rho_{\text {red }}(t)\right| y_{1}\right\rangle= & \int d x_{0} \int d y_{0} \int_{x_{0}}^{x_{1}} \mathcal{D} x \\
& \times \int_{y_{0}}^{y_{1}} \mathcal{D} y e^{i S_{0}(x)-i S_{0}(y)} \\
& \times F[x, y]\left\langle x_{0}\left|\rho_{\text {sys }}(0)\right| y_{0}\right\rangle,
\end{aligned}
$$

where we have introduced the influence functional

$$
\begin{aligned}
F[x, y] & =\operatorname{Tr}_{\text {res }}\left[U(x, t) \rho_{\text {res }}(0) U^{\dagger}(y, t)\right] \\
& \equiv\left\langle U^{\dagger}(y, t) U(x, t)\right\rangle .
\end{aligned}
$$

We have assumed that the system and reservoir decouples at $t=0$,

$$
\rho(0)=\rho_{\text {sys }}(0) \otimes \rho_{\text {res }}(0) .
$$

This decoupling is unphysical, but after a very short time, the system and reservoir are thoroughly mixed, and all memory of the initial condition has vanished.

Thus, the influence functional couples the paths mov- 
ing forward and backward in time. We are now in position to define the effective action for the system, where the reservoir influence is included, simply as

$$
e^{i \Delta S_{\text {eff }}(x, y)}=F[x, y]=\left\langle U_{\gamma}\left(0^{\prime}, 0\right)\right\rangle
$$

Here, we have introduced the contour $\gamma$, see Fig. 3, and the time-evolution operator on $\gamma$,

$$
U_{\gamma}\left(0^{\prime}, 0\right)=T_{\gamma} e^{-i \int_{\gamma} d \tau H_{\mathrm{res}}+H_{\mathrm{int}}\left(x_{\gamma}(\tau)\right)},
$$

where $x_{\gamma}(\tau)$ is a combined path, which is equal to $x(\tau)$ when $\tau$ is on the upper part of the contour and equal to $y(\tau)$ on the lower part. To obtain $\Delta S_{\text {eff }}$, we use a mathematical standard procedure (Linked Cluster), where we replace $V$ by $\lambda V$ and then use the operator identity,

$$
\frac{d}{d \lambda} U_{\gamma}\left(0^{\prime}, 0\right)=-i \int_{\gamma} d \tau U_{\gamma}\left(0^{\prime}, \tau\right) V(\tau) U_{\gamma}(\tau, 0) .
$$

Taking \langle\rangle , we get a first order differential equation in $\lambda$ for $F_{\lambda}(x, y)$, where $F_{0}(x, y)=1$. Solving this and taking $\lambda=1$, we see

$$
F(x, y)=\left\langle U_{\gamma}\left(0^{\prime}, 0\right)\right\rangle=e^{-i \int_{0}^{1} d \lambda \int_{\gamma} d \tau \mathcal{F}_{\lambda}(\tau)},
$$

with

$$
\mathcal{F}_{\lambda}(\tau) \equiv \frac{\left\langle U_{\gamma}\left(0^{\prime}, \tau\right) V(\tau) U_{\gamma}(\tau, 0)\right\rangle}{\left\langle U_{\gamma}\left(0^{\prime}, 0\right)\right\rangle}
$$

and we finally obtain the desired expression for the action,

$$
\Delta S_{\text {eff }}(x, y)=-\int_{0}^{1} d \lambda \int_{\gamma} d \tau \mathcal{F}_{\lambda}(\tau)
$$

This can be written in terms of the contour Green's functions $G_{k k^{\prime}}^{\gamma}$, with $T_{\gamma}$ being the contour time-ordering operator:

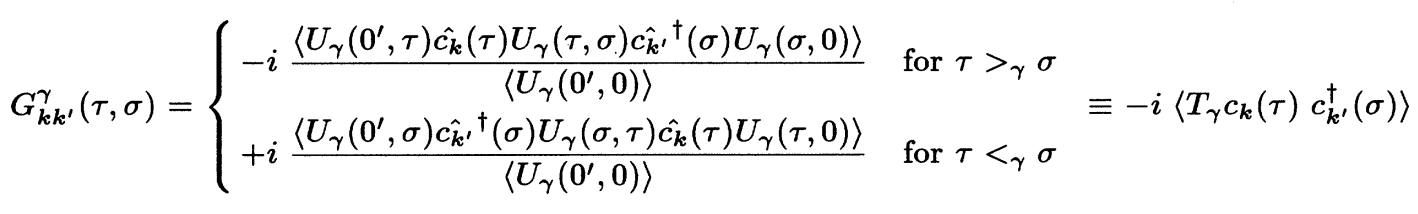

as

$$
\mathcal{F}_{\lambda}(\tau)=-i \sum_{k k^{\prime}} G_{k k^{\prime}}^{\gamma}\left(\tau, \tau_{+}\right) V_{k k^{\prime}}\left(X_{\gamma}(\tau)\right)
$$

Following the Keldysh formalism, ${ }^{20}$ we can represent the contour Green's function by the real-time axis, using $2 \times 2$ matrices with the usual real-time Green's functions as elements. The first matrix index decides whether the first argument is on the upper ( $x$-part) or the lower $(y$ part) branch of $\gamma$, and likewise for the second argument and matrix index. Defining

$$
\mathbf{G}_{k k^{\prime}}\left(t, t^{\prime}\right)=\left(\begin{array}{ll}
G_{k k^{\prime}}\left(t, t^{\prime}\right) & G_{k k^{\prime}}^{<}\left(t, t^{\prime}\right) \\
G_{k k^{\prime}}^{>}\left(t, t^{\prime}\right) & \bar{G}_{k k^{\prime}}\left(t, t^{\prime}\right)
\end{array}\right)
$$

and

$$
\mathbf{V}_{k k^{\prime}}(t)=\left(\begin{array}{cc}
V_{k k^{\prime}}(x(t)) & 0 \\
0 & -V_{k k^{\prime}}(y(t))
\end{array}\right)
$$

we can write the effective action,

$$
\Delta S_{\mathrm{eff}}(x, y)=i \int_{0}^{1} d \lambda \int_{0}^{t} d t^{\prime} \sum_{k k^{\prime}} \operatorname{Tr}\left[\mathbf{G}_{k k^{\prime}}\left(t^{\prime}, t^{\prime}\right) \mathbf{V}_{k k^{\prime}}\left(t^{\prime}\right)\right]
$$

where $\mathbf{G}$ satisfy the Dyson equation

$$
\begin{aligned}
\mathbf{G}_{k k^{\prime}}\left(t_{2}-t_{1}\right)= & \delta_{k k^{\prime}} \mathbf{G}_{k}^{(0)}\left(t_{2}-t_{1}\right) \\
& +\lambda \sum_{q} \int_{0}^{t} d t^{\prime} \mathbf{G}_{k}^{(0)}\left(t_{2}\right. \\
& \left.-t^{\prime}\right) \mathbf{V}_{k q}\left(t^{\prime}\right) \mathbf{G}_{q k^{\prime}}\left(t^{\prime}-t_{1}\right)
\end{aligned}
$$

Here, $\mathbf{G}^{(0)}$ is

$$
\mathbf{G}_{k}^{(0)}\left(t, t^{\prime}\right)=i e^{-i \epsilon_{k}\left(t-t^{\prime}\right)}\left[n_{F}\left(\epsilon_{k}\right)\left(\begin{array}{ll}
1 & 1 \\
1 & 1
\end{array}\right)-\left(\begin{array}{cc}
\theta\left(t-t^{\prime}\right) & 0 \\
1 & \theta\left(t^{\prime}-t\right)
\end{array}\right)\right]
$$

Iterating (A18), we can write the effective action as a sum of terms of the form

$$
\begin{aligned}
\Delta S_{\text {eff }}^{(n)}(x, y)= & \frac{i}{n} \sum_{k_{n}, \cdots, k_{1}} \int_{0}^{t} d t_{n} \cdots \int_{0}^{t} d t_{1} \operatorname{Tr}\left[\mathbf{G}_{k_{n}}^{(0)}\left(t_{1}, t_{n}\right) \mathbf{V}_{k_{n} k_{n-1}}\left(t_{n}\right) \mathbf{G}_{k_{n-1}}^{(0)}\right. \\
& \left.\times\left(t_{n}, t_{n-1}\right) \cdots \mathbf{G}_{k_{1}}^{(0)}\left(t_{2}, t_{1}\right) \mathbf{V}_{k_{1} k_{n}}\left(t_{1}\right)\right] .
\end{aligned}
$$

\section{The effective potential}

Now, let us find the time-local part of $\Delta S_{\text {eff }}$, which does not depend on the time derivatives of the adsorbate motion. The important step in the derivation is the partial integration, 


$$
\begin{aligned}
\int_{0}^{t} d t_{i} e^{i\left(\epsilon_{k_{i}}-\epsilon_{k_{i-1}}\right) t_{i}} \theta\left(t_{i}-t_{i-1}\right) V_{k_{i} k_{i-1}}\left(x\left(t_{i}\right)\right) \cdots \sim & -\int_{0}^{t} d t_{i} \frac{e^{i\left(\epsilon_{k_{i}}-\epsilon_{k_{i-1}}\right) t_{i}}}{i\left(\epsilon_{k_{i}}-\epsilon_{k_{i-1}}\right)}\left[\delta\left(t_{i}-t_{i-1}\right) V_{k_{i} k_{i-1}}\left(x\left(t_{i}\right)\right)\right. \\
& \left.+\theta\left(t_{i}-t_{i-1}\right) V_{k_{i} k_{i-1}}^{\prime}\left(x\left(t_{i}\right)\right) \dot{x}\left(t_{i}\right)\right] \cdots
\end{aligned}
$$

where we neglect the end-point contribution. To obtain the time local contribution, we must use the part of $\Delta S_{\text {eff }}^{(n+1)}$, with precise $n \theta$ functions (and therefore one $n_{F}$ ). The $n-1 \theta$ parts of the $n \mathbf{G}^{(0)}$ 's in (A20) can be picked in $n$ ways, thus giving a factor of $n$. We identify the desired part as

$$
\begin{aligned}
& (-1)^{n} \sum_{k_{n}, \ldots, k_{1}} n_{F}\left(\epsilon_{k_{n}}\right) \int_{0}^{t} d t_{n} \cdots \int_{0}^{t} d t_{1} i^{n-1} e^{i\left[t_{n}\left(\epsilon_{k_{n}}-\epsilon_{k_{n-1}}\right)+\cdots+t_{1}\left(\epsilon_{k_{1}}-\epsilon_{k_{n}}\right)\right]} \\
& \times\left[V_{k_{1} k_{n}}\left(x\left(t_{1}\right)\right)\left\{V_{k_{n} k_{n-1}}\left(x\left(t_{n}\right)\right) \cdots V_{k_{2} k_{1}}\left(x\left(t_{2}\right)\right) \theta\left(t_{n}-t_{n-1}\right) \cdots \theta\left(t_{2}-t_{1}\right)\right\}\right. \\
& \left.-V_{k_{1} k_{n}}\left(y\left(t_{1}\right)\right)\left\{V_{k_{n} k_{n-1}}\left(y\left(t_{n}\right)\right) \cdots V_{k_{2} k_{1}}\left(y\left(t_{2}\right)\right) \theta\left(t_{n}-t_{n-1}\right) \cdots \theta\left(t_{2}-t_{1}\right)\right\}\right] .
\end{aligned}
$$

Now, we do the $n-1$ partial integrations (keeping the last integration) and end up with the time-local and velocityindependent part of $\Delta S_{\mathrm{eff}}^{(n)}$ :

$$
\begin{aligned}
& -\sum_{k_{n}, . ., k_{1}} n_{F}\left(\epsilon_{k_{n}}\right) \int_{0}^{t} d t_{1} V_{k_{1} k_{n}}\left(x\left(t_{1}\right)\right) \\
& \times \prod_{i=1}^{n-1} \frac{V_{k_{i+1} k_{i}}\left(x\left(t_{1}\right)\right)}{\epsilon_{k_{n}}-\epsilon_{k_{i}}} \\
& -V_{k_{1} k_{n}}\left(y\left(t_{1}\right)\right) \prod_{i=1}^{n-1} \frac{V_{k_{i+1} k_{i}}\left(y\left(t_{1}\right)\right)}{\epsilon_{k_{n}}-\epsilon_{k_{i}}}
\end{aligned}
$$

This result we compare to the standard Linked Cluster calculation of the equilibrium free energy using the Matsubara technique: ${ }^{21}$

$$
\begin{aligned}
F= & F_{0}-\frac{1}{\beta} \sum_{l=1}^{\infty} \frac{(-1)^{l}}{l} \\
& \times \int_{0}^{\beta} d \tau_{n} \cdots \int_{0}^{\beta} d \tau_{1}\left\langle T_{\tau} V\left(\tau_{l}\right) \cdots V\left(\tau_{1}\right)\right\rangle .
\end{aligned}
$$

To get the $n$th order correction, we must evaluate the closed loop diagram shown in Fig. 9, giving

$$
\begin{aligned}
F^{(n)}= & \frac{1}{n} \sum_{k_{n}, \ldots, k_{1}} \frac{1}{\beta} \sum_{i p_{n}} V_{k_{1} k_{n}} \mathcal{G}^{(0)}\left(i p_{n}, \epsilon_{k_{n}}\right) \\
& \times V_{k_{n} k_{n-1}} \mathcal{G}^{(0)}\left(i p_{n}, \epsilon_{k_{n-1}}\right) \cdots V_{k_{2} k_{1}} \mathcal{G}^{(0)} \\
& \times\left(i p_{n}, \epsilon_{k_{1}}\right)
\end{aligned}
$$

doing the frequency summation, ${ }^{21}$ we get precisely the potential corresponding to the contribution to the action given by (A23) as could be expected. This free energy will, in general, give a temperature-dependent renormalization of the potential $V_{\text {eff }}$. From this point on, the potential energy term contributing to the action $S_{0}$ is taken to be the fully renormalized and temperature dependent $V_{\text {eff }}$ just derived.

To get a more convenient expression for the free energy, $F$, one can, e.g., use the standard formula,

$$
\begin{aligned}
F(x) & =-\frac{1}{\beta} \ln (Z) \\
& =-\frac{1}{\beta} \int_{-\infty}^{\infty} \ln \left(1+e^{-\beta(\omega-\mu)}\right) A(\omega, x) \frac{d \omega}{2 \pi} \\
& =-\frac{1}{\pi} \int_{-\infty}^{\infty} d \epsilon n_{F}(\epsilon) \delta(\epsilon, x)
\end{aligned}
$$

Here the spectral function, $A$, and the phase shift, $\delta$, is defined in (A47) and (A50), respectively, and we have used partial integration.

\section{The temperature-dependent friction}

We will now outline the derivation of the Langevin equation from (8) and, in particular, find the relevant friction coefficient, $\eta$. The reduced density matrix is a path integral over pairs of paths $x$ and $y$. As mentioned in the main text, we shall find that only pairs of paths that are sufficiently close will contribute significantly. It is the

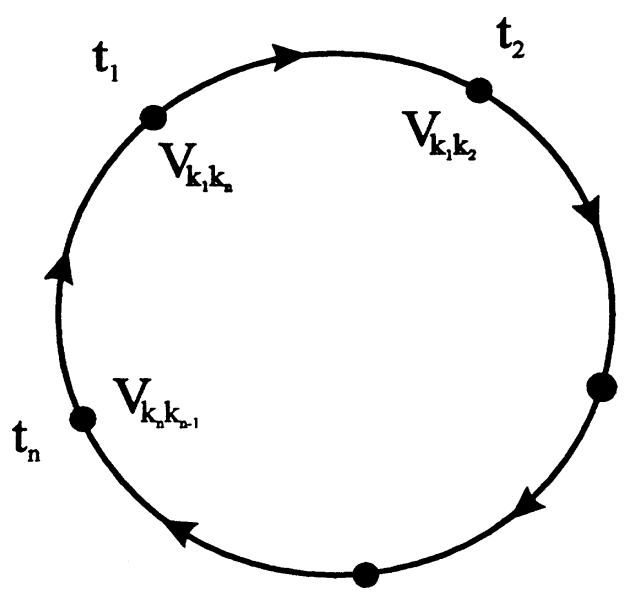

FIG. 9. The $n$th order diagram with Matsubara Green's functions. 
influence functional which suppress very deviating pairs of paths. The logic of the derivation goes as follows. We first assume, that the paths $x$ and $y$ are so close that an expansion to second order in the deviation $\xi=x-y$ of the Hamiltonian is adequate. With this approximation, we calculate the influence functional. Knowing the influence functional, we can then verify that our initial assumption holds.

Let us consider a pair of paths $x$ and $y$, which have made it to a region close to a point $Q_{0}$ at time $t_{0}$. We will now evaluate the contribution to $\Delta S_{\text {eff }}$ from time interval $\left(t_{0}, t_{0}+\delta\right)$. We will take $\delta$ to be so short that the following expansion of the full interaction part of the Hamiltonian is allowed:

$$
\begin{aligned}
H_{\mathrm{int}}(x) & =H_{\mathrm{int}}\left(Q_{0}\right)+\left(x-Q_{0}\right) \sum_{k k^{\prime}} V_{k k^{\prime}}^{\prime} c_{k}^{\dagger} c_{k^{\prime}}, \\
V_{k k^{\prime}}^{\prime} & \equiv \frac{\partial V_{k k^{\prime}}}{\partial x}\left(Q_{0}\right)
\end{aligned}
$$

Consider the 2 order term (the 1 order term is included in $V_{\text {eff }}$ ) of (A17) in $V_{\boldsymbol{k}^{\prime}}^{\prime}$, with $\mathbf{G}$ being the Green's function matrix corresponding to $H_{\text {int }}\left(Q_{0}\right)$ :

$$
\begin{aligned}
\Delta S_{\mathrm{eff}}^{(2)}(x, y)= & \frac{i}{2} \int_{0}^{\delta} d t_{1} \int_{0}^{\delta} d t_{2} \sum_{\substack{k_{1}, k_{2} \\
k_{3}, k_{4}}} V_{k_{2} k_{3}}^{\prime} V_{k_{4} k_{1}}^{\prime} \\
& \times \operatorname{Tr}\left[\mathbf{G}_{k_{1} k_{2}}\left(t_{1}, t_{2}\right) \mathbf{X}\left(t_{2}\right)\right. \\
& \left.\times \mathbf{G}_{k_{3} k_{4}}\left(t_{2}, t_{1}\right) \mathbf{X}\left(t_{1}\right)\right]
\end{aligned}
$$

where we have defined,

$$
\mathbf{X}\left(t^{\prime}\right) \equiv\left(\begin{array}{cc}
x\left(t^{\prime}\right)-Q_{0} & 0 \\
0 & -y\left(t^{\prime}\right)+Q_{0}
\end{array}\right)
$$

We write $\mathbf{G}$ in the spectral representation:

$$
\mathbf{G}_{k_{1} k_{2}}^{(0)}\left(t_{2}-t_{1}\right)=\int_{-\infty}^{\infty} \frac{d \omega}{2 \pi} e^{-i \omega\left(t_{2}-t_{1}\right)} R_{k_{1} k_{2}}(\omega) i\left(\begin{array}{cc}
n_{F}(\omega)-\theta\left(t_{2}-t_{1}\right) & n_{F}(\omega) \\
n_{F}(\omega)-1 & n_{F}(\omega)-\theta\left(t_{1}-t_{2}\right)
\end{array}\right)
$$

with $R_{k k^{\prime}}(\omega)$ being the spectral functions,

$$
R_{k k^{\prime}}(\omega)=-2 \operatorname{Im}\left[G_{k k^{\prime}}^{\mathrm{ret}}(\omega)\right] .
$$

Defining, a weighted electron-hole pair density of states,

$$
\Gamma\left(\omega_{1}, \omega_{2}\right) \equiv \sum_{\substack{k_{1}, k_{2} \\ k_{3}, k_{4}}} R_{k_{1} k_{2}}\left(\omega_{1}\right) V_{k_{2} k_{3}} R_{k_{3} k_{4}}\left(\omega_{2}\right) V_{k_{4} k_{1}}
$$

we can write,

$$
\begin{aligned}
\Delta S_{\mathrm{eff}}^{(2)}(x, y)= & -\frac{i}{2} \int_{0}^{\delta} d t_{1} \int_{0}^{\delta} d t_{2} \int_{-\infty}^{\infty} \frac{d \omega_{1}}{2 \pi} \int_{-\infty}^{\infty} \frac{d \omega_{1}}{2 \pi} \Gamma\left(\omega_{1}, \omega_{2}\right) e^{i\left(\omega_{1}-\omega_{2}\right)\left(t_{2}-t_{1}\right)} \\
& \times \operatorname{Tr}\left[\begin{array}{cc}
n_{F}\left(\omega_{1}\right)-\theta\left(t_{1}-t_{2}\right) & n_{F}\left(\omega_{1}\right) \\
n_{F}\left(\omega_{1}\right)-1 & n_{F}\left(\omega_{1}\right)-\theta\left(t_{2}-t_{1}\right)
\end{array}\right)\left(\begin{array}{cc}
x\left(t_{2}\right)-Q_{0} & 0 \\
0 & -\left[y\left(t_{2}\right)-Q_{0}\right]
\end{array}\right) \\
& \left.\times\left(\begin{array}{cc}
n_{F}\left(\omega_{2}\right)-\theta\left(t_{2}-t_{1}\right) & n_{F}\left(\omega_{2}\right) \\
n_{F}\left(\omega_{2}\right)-1 & n_{F}\left(\omega_{2}\right)-\theta\left(t_{1}-t_{2}\right)
\end{array}\right)\left(\begin{array}{cc}
x\left(t_{1}\right)-Q_{0} & 0 \\
0 & -\left[y\left(t_{1}\right)-Q_{0}\right]
\end{array}\right)\right]
\end{aligned}
$$

Trivial calculations using the identity,

$$
\left[n_{F}(x)-\theta(t)\right]\left[n_{F}(y)-\theta(-t)\right]=\frac{1}{2}\left[\operatorname{sign}(t)+\operatorname{coth}\left(\frac{\beta(x-y)}{2}\right)\right]\left[n_{F}(x)-n_{F}(y)\right]
$$

yields the real part:

$$
\begin{aligned}
& -\int_{0}^{\delta} d t_{2} \int_{0}^{t_{2}} d t_{1} \int_{-\infty}^{\infty} \frac{d \omega}{2 \pi} \Lambda(\omega) \cos \left[\omega\left(t_{2}-t_{1}\right)\right] \frac{1}{2}\left[\dot{x}\left(t_{1}\right)\right. \\
& \left.+\dot{y}\left(t_{1}\right)\right]\left[x\left(t_{2}\right)-y\left(t_{2}\right)\right]
\end{aligned}
$$

where we have neglected the time-local term already included in $V_{\text {eff }}$, and defined

$$
\begin{aligned}
\Lambda(\omega)= & -\frac{1}{\omega} \int_{-\infty}^{\infty} \frac{d \omega_{1}}{2 \pi} \int_{-\infty}^{\infty} d \omega_{2} \delta\left[\omega-\left(\omega_{2}\right.\right. \\
& \left.\left.-\omega_{1}\right)\right] \Gamma\left(\omega_{1}, \omega_{2}\right)\left[n_{F}\left(\omega_{2}\right)-n_{F}\left(\omega_{1}\right)\right] \\
= & -\frac{1}{\omega} \int_{-\infty}^{\infty} \frac{d \omega_{1}}{2 \pi} \Gamma\left(\omega_{1}, \omega+\omega_{1}\right)\left[n_{F}\left(\omega+\omega_{1}\right)\right. \\
& \left.-n_{F}\left(\omega_{1}\right)\right] .
\end{aligned}
$$

The function $\Lambda$ plays the central role in our calculation, 


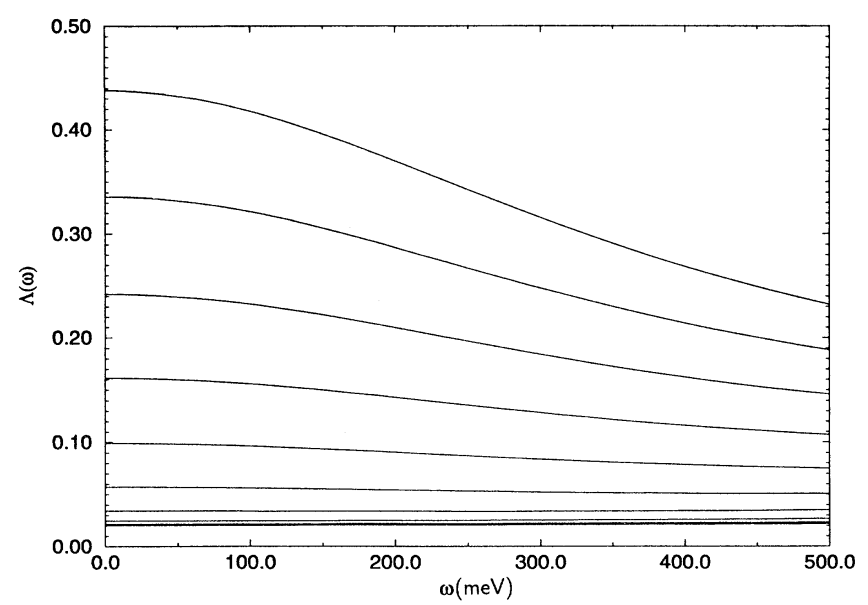

FIG. 10. $\Lambda$ in a.u. ${ }^{-2}$ for temperatures from $5500 \mathrm{~K}$ (upper) in steps of $500 \mathrm{~K}$ to $1000 \mathrm{~K}$ (lower). The strong temperature dependence of $\eta=\Lambda(0) / 2$ is seen. The parameters used are $\epsilon_{\infty}=5.0 \mathrm{eV}, C=3.0 \mathrm{eV}, \Delta_{0}=0.2 \mathrm{eV}, \alpha=0.5$ a.u., and $\beta=1.0$ a.u., corresponding to a peak electron temperature of $5500 \mathrm{~K}$.

and it is plotted for different temperatures in Fig. 10 for realistic parameters using (22). It varies, for the relevant temperatures, on a scale essentially given by $\Delta$, so since $\Lambda$ varies on a corresponding time scale much shorter than the classical time scale, we can replace the Fourier transform of $\Lambda(\omega)$ by $\Lambda(0) \delta\left(t_{1}-t_{2}\right)$.

We have that

$$
\Lambda(0)=\int_{-\infty}^{\infty} \frac{d \omega}{2 \pi} \Gamma(\omega, \omega)\left(-\frac{\partial n_{F}}{\partial \omega}(\omega)\right)
$$

and get

$$
\begin{aligned}
\operatorname{Re} \Delta S_{\mathrm{eff}}^{(2)}(x, y)= & -\frac{\Lambda(0)}{2} \int_{0}^{\delta} d t_{1} \frac{1}{2}\left[\dot{x}\left(t_{1}\right)\right. \\
& \left.+\dot{y}\left(t_{1}\right)\right]\left[x\left(t_{1}\right)-y\left(t_{1}\right)\right]
\end{aligned}
$$

Also using the $\Lambda$ function, we can write the imaginary part of $\Delta S_{\text {eff }}^{(0)}$ as,

$$
\begin{aligned}
\operatorname{Im} S_{\text {eff }}(x, y)= & \int_{0}^{\delta} d t_{1} \int_{0}^{\delta} d t_{2}\left[x\left(t_{1}\right)-y\left(t_{1}\right)\right] K\left(t_{1}\right. \\
& \left.-t_{2}\right)\left[x\left(t_{2}\right)-y\left(t_{2}\right)\right],
\end{aligned}
$$

where the kernel is

$$
\begin{aligned}
K(t) & =\int_{0}^{\infty} \frac{d \omega}{2 \pi} \omega \Lambda(\omega) \operatorname{coth}\left(\frac{\omega}{2 k T}\right) \cos (\omega t), \\
& \approx \frac{\Lambda(0)}{2} \int_{-\infty}^{\infty} \frac{d \omega}{2 \pi} e^{-\omega / \Omega} \omega \operatorname{coth}\left(\frac{\omega}{2 k T}\right) \cos (\omega t) .
\end{aligned}
$$

We neglect here the weak change in $\Lambda$ on the $k T$ scale (see Fig. 10). Furthermore, a high-energy cutoff $\Omega\left(\approx \epsilon_{F}\right)$ on the excitations of electron-hole pairs is introduced.

We shall soon see that $\Lambda(0) / 2=\eta\left(Q_{0}\right)$ can be identified as the usual friction coefficient entering the Langevin equation. First, however, let us show that paths that deviate strongly are sufficiently suppressed, thereby justifying our initial expansions (A28) and (A29). Consider a fluctuation in the paths where $x$ and $y$ deviates by $r_{0} \sim V\left(Q_{0}\right) / V^{\prime}\left(Q_{0}\right)$, for a time $\Delta t . r_{0}$ is the characteristic length scale for the variation of $V$. From (A40a) we get that such a pair of paths is damped by a factor proportional to

$$
e^{-\frac{1}{2} A(\Delta t) r_{0}^{2}}
$$

where

$$
\begin{aligned}
A(\Delta t) & \approx 2 \Lambda(0) \int_{0}^{\infty} \frac{d \omega}{2 \pi} e^{-\omega / \Omega} \frac{1}{\omega} \operatorname{coth}\left(\frac{\omega}{2 k T}\right)[1-\cos (\omega \Delta t)] \\
& =\frac{\Lambda(0)}{2 \pi} \ln \left[\frac{1+(\Delta t \Omega)^{2}}{|\Gamma(1+i \Delta t \Omega+k T / \Omega)|^{4}}\left(\frac{k T}{\Omega} \Gamma\left(\frac{k T}{\Omega}\right)\right)^{4}\right] \\
& \approx \frac{\Lambda(0)}{\pi}\left(\frac{1}{2} \ln \left[1+(\Omega \Delta t)^{2}\right]+\ln \left[\frac{1}{\pi \Delta t k T} \sinh (\pi \Delta t k T)\right]\right) .
\end{aligned}
$$

In the last approximation, we have assumed that $k T \ll \Omega$. Now, the typical duration of a fluctuation, $\Delta t$, is estimated by setting the exponent in (A41a) equal to 1 . We have for realistic parameters (see Fig. 10) that $\Lambda(0) r_{0}^{2} \sim$ $\Lambda(0)(\operatorname{Min}(\alpha, \beta))^{2}$ lies in the range $0.01-0.5$ for temperatures in the range $1000-5500 \mathrm{~K}$, so an estimate of the largest size of $\delta$ is approximately determined by the equation

$$
\frac{1}{2 \pi}\left(\ln \left[1+(\Delta t \Omega)^{2}\right]+2 \ln \left[\frac{1}{\pi \Delta t k T} \sinh (\pi \Delta t k T)\right]\right) \sim[2 ; 100] .
$$

We can determine the upper limits on $\Delta t$ (taking $\Omega=0$ ) to be less than about 0.1 fs for a temperature of $5500 \mathrm{~K}$, which increases to about $15 \mathrm{fs}$ for $1000 \mathrm{~K}$. So we see that these times are considerably shorter than all other times in the problem. Indeed paths $x$ and $y$ follow each other quite closely, justifying our basic assumption.

To reach the Langevin equation, we follow $\mathrm{Schmid}^{17}$ and change variables in the path integral,

$$
Q(\tau)=\frac{1}{2}[x(\tau)+y(\tau)], \xi(\tau)=x(\tau)-y(\tau)
$$




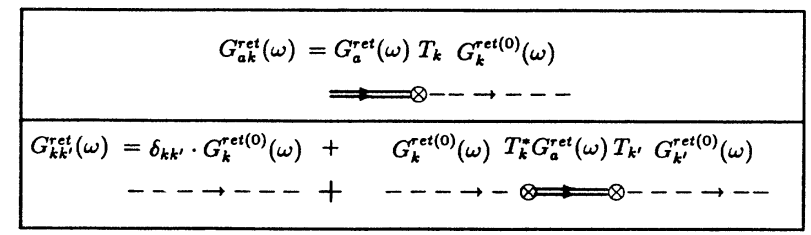

FIG. 11. The spectral function is found from the retarded Green's functions shown diagramatically.

and write the total path integral (inserting $\hbar$ ),

$$
\int \mathcal{D} Q \int \mathcal{D} \xi e^{\frac{i}{\hbar} \int_{0}^{t} d \tau\left[M \ddot{Q}(\tau)+V_{\mathrm{eff}}^{\prime}(Q)+\eta(Q) \dot{Q}(\tau)\right] \xi(\tau)-\frac{1}{\hbar} \int_{0}^{t} d \tau \int_{0}^{t} d \sigma \xi(\tau) K(\tau-\sigma) \xi(\sigma)} .
$$

We have here made the expansion of $V_{\text {eff }}$,

$$
V_{\mathrm{eff}}(x)-V_{\mathrm{eff}}(y) \approx V_{\mathrm{eff}}^{\prime}(Q) \xi
$$

which is justified in the same way as above.

A probabilistic interpretation of this path integral is equivalent to the Langevin equation with the force correlation function given by $K$. For further discussion of this point, we refer to Schmid. ${ }^{17}$ In the high temperature limit, where the thermal correlation time $\mathrm{e}^{22}$ determined from $K, \hbar / 2 \pi k T$, is much smaller than all the time scales of motion, we get the familiar result of the time-local fluctuating force,

$$
\langle f(t) f(0)\rangle=K(t) \sim 2 \eta k T \delta(t) .
$$

The correlation time is $1.27 \mathrm{ps} / \mathrm{K}$, so it is about 1 fs for a temperature of $1000 \mathrm{~K}$, and we can immediately assume a $\delta$ correlation. It is clear by comparing the real part of $S_{\text {eff }}$ in equation (A39) that we can identify $\eta$ with $\Lambda(0) / 2$.

When applying these results, one should keep in mind that $\Lambda(0)$ is a quantity that is specific to the position at which the particle finds itself at a given time $t_{0}$. We, therefore, have arrived at a position- and temperature-dependent friction coefficient.

Finally, we calculate $\eta$ in terms of the model parameters. First, we find the spectral functions in terms of $\Delta$ and $\mathcal{H}$ and

$$
R_{a a}(\omega) \equiv A\left(\omega, Q_{0}\right)=\frac{2 \Delta\left(\omega, Q_{0}\right)}{\left[\omega-\varepsilon\left(Q_{0}\right)-\mathcal{H}\left(\omega, Q_{0}\right)\right]^{2}+\Delta\left(\omega, Q_{0}\right)^{2}}
$$

where we have written the $Q_{0}$ dependence explicitly. From Fig. 11, we see that $\left(a \neq\left\{k, k^{\prime}\right\}\right)$,

and

$$
\begin{aligned}
R_{a k}\left(\omega, Q_{0}\right) & =-2 \operatorname{Im}\left[\int_{-\infty}^{\infty} \frac{d \omega^{\prime}}{2 \pi} \frac{A\left(\omega^{\prime}, Q_{0}\right)}{\omega-\omega^{\prime}+i \delta} T_{k}\left(Q_{0}\right) \frac{1}{\omega-\epsilon_{k}+i \delta}\right] \\
& =T_{k}\left(Q_{0}\right)\left(A\left(\omega, Q_{0}\right) P \frac{1}{\omega-\epsilon_{k}}+A\left(\omega, Q_{0}\right) \frac{\omega-\epsilon_{a}-\mathcal{H}\left(\omega, Q_{0}\right)}{\Delta\left(\omega, Q_{0}\right)} \pi \delta\left(\omega-\epsilon_{k}\right)\right),
\end{aligned}
$$

$$
R_{k k^{\prime}}\left(\omega, Q_{0}\right)=\delta_{k k^{\prime}} 2 \pi \delta\left(\omega-\epsilon_{k}\right)-2 \operatorname{Im}\left[T_{k}\left(Q_{0}\right) T_{k^{\prime}}\left(Q_{0}\right) \int_{-\infty}^{\infty} \frac{d \omega^{\prime}}{2 \pi} \frac{A\left(\omega^{\prime}, Q_{0}\right)}{\omega-\omega^{\prime}+i \delta} \frac{1}{\omega-\epsilon_{k}+i \delta} \frac{1}{\omega-\epsilon_{k^{\prime}}+i \delta}\right]
$$

Inserting these in the expression for $\Gamma\left(\omega, \omega, Q_{0}\right)$ (A33) we get after some trivial manipulations, the space- and temperature-dependent friction coefficient,

$$
\begin{aligned}
\eta\left(Q_{0}, T\right)=\frac{1}{2} \Lambda(0)= & \lim _{\omega \rightarrow 0} \frac{-1}{2 \omega} \int_{-\infty}^{\infty} \frac{d \omega_{1}}{2 \pi} \Gamma\left(\omega_{1}, \omega+\omega_{1}\right)\left[n_{F}\left(\omega+\omega_{1}\right)-n_{F}\left(\omega_{1}\right)\right] \\
= & \frac{1}{4 \pi} \int_{-\infty}^{\infty} d \omega \Gamma(\omega, \omega)\left(-\frac{d n_{F}}{d \omega}\right) \\
= & \frac{1}{4 \pi} \int_{-\infty}^{\infty} d \omega\left[A\left(\frac{\partial\left(\varepsilon_{a}+\mathcal{H}\right)}{\partial x}+\frac{\left(\omega-\epsilon_{a}-\mathcal{H}\right)}{\Delta} \frac{\partial \Delta}{\partial x}\right)\right]^{2}\left(\omega, Q_{0}\right)\left(-\frac{d n_{F}}{d \omega}(\omega)\right) \\
& +\frac{1}{4 \pi} \int_{-\infty}^{\infty} d \omega\left[4 \pi A \sum_{k}\left|T_{k}^{\prime}\right|^{2} \delta\left(\omega-\epsilon_{k}\right)-4 \frac{A}{\Delta}\left(\frac{1}{2} \frac{\partial \Delta}{\partial x}\right)^{2}\right]\left(\omega, Q_{0}\right)\left(-\frac{d n_{F}}{d \omega}(\omega)\right)
\end{aligned}
$$


Introducing the phase shift,

$$
\delta(\omega, x)=\frac{\pi}{2}-\arctan \left(\frac{\varepsilon_{a}(x)+\mathcal{H}(\omega, x)-\omega}{\Delta(\omega, x)}\right)
$$

and using the identity

$$
\sin ^{2}(\delta)=\frac{A}{2} \Delta
$$

we observe that we can write the friction as

$$
\begin{aligned}
\eta\left(Q_{0}, T\right)= & \frac{1}{\pi} \int_{-\infty}^{\infty} d \omega\left(\frac{\partial \delta}{\partial x}\left(\omega, Q_{0}\right)\right)^{2}\left(-\frac{d n_{F}}{d \omega}(\omega)\right) \\
& +\frac{1}{\pi} \int_{-\infty}^{\infty} d \omega 2 \sin ^{2}(\delta)\left[\frac{\sum_{k}\left|T_{k}^{\prime}\left(Q_{0}\right)\right|^{2} \delta\left(\omega-\epsilon_{k}\right)}{\sum_{k}\left|T_{k}\left(Q_{0}\right)\right|^{2} \delta\left(\omega-\epsilon_{k}\right)}-\left(\frac{1}{2} \frac{\partial \Delta}{\partial x}\right)^{2}\right](\omega)\left(-\frac{d n_{F}}{d \omega}(\omega)\right) .
\end{aligned}
$$

This friction coefficient is exactly equal to the result obtained by Nourtier, ${ }^{8}$ using a different approach. We can neglect the last term (A49d, A52b), if we assume the same spatial decay for $T_{k}, T_{k}^{\prime} \approx c T_{k}$, for the important $k^{\prime} s$ with $\epsilon_{k}$ within the $k T$ range of $\epsilon_{F}$.

${ }^{1}$ J.A. Prybyla, T.F. Heinz, M.M.T. Loy, and J.H. Glownia, Phys. Rev. Lett. 64, 1537 (1990); J.A. Misewich, A. Kalamarides, T.F. Heinz, U. Hofer, and M.M.T. Loy, J. Chem. Phys. 100, 736 (1994).

2 J.A. Prybyla, H.W.K. Tom, and G.D. Aumiller, Phys. Rev. Lett. 68, 503 (1992).

${ }^{3}$ F. Budde, T.F. Heinz, M.M.T. Loy, J.A. Misewich, F. de Rougemont, and H. Zacharias, Phys. Rev. Lett. 66, 3024 (1991).

4 P.B. Allen, Phys. Rev. Lett. 59, 1460 (1987).

${ }^{5}$ D.M. Newns, Surf. Sci. 171, 600 (1986); H. Kasai and A. Okiji, ibid. 225, L33 (1990).

${ }^{6}$ F. Budde, T.F. Heinz, A. Kalamarides, M.M.T. Loy, and J.A. Misewich, Surf. Sci. 283, 143 (1993).

${ }^{7}$ B.N.J. Persson and M. Persson, Solid State Commun. 36, 175 (1980); Phys. Rev. Lett. 54, 2119 (1985).

${ }^{8}$ K.P. Bohnen, M. Kiwi, and H. Suhl, Phys. Rev. Lett. 34, 1512 (1975); A. Nourtier, J. Phys. (Paris) 38, 479 (1977).

${ }^{9}$ A. Yoshimori and J.L. Motchane, J. Phys. Soc. Jpn. 51, 1826 (1982); A. Okiji and H. Kasai, Surf. Sci. B 188, L717 (1987).

10 J.C. Tully, M. Gomez, and M. Head Gordon, J. Vac. Sci. Technol. 11, 1914 (1993); J.C. Tully and M. Head Gordon, Phys. Rev. B 46, 1853 (1992).

${ }^{11}$ D.M. Newns, T.F. Heinz, and J.A. Misewich, Prog. Theor. Phys. 106, 411 (1991).

12 J.A. Misewich, T.F. Heinz, and D.M. Newns, Phys. Rev.
Lett. 68, 25 (1992); 68, 3737 (1992).

${ }^{13}$ D.M. Newns, T.F. Heinz, J.A. Misewich, M. Brandbyge, and P. Hedegård (unpublished).

${ }^{14}$ R.P. Feynman and F.L. Vernon, Ann. Phys. (N.Y.) 24, 118, (1963)

${ }^{15}$ R.P. Feynman and A.R. Hibbs, Quantum Mechanics and Path Integrals (McGraw-Hill, New York, 1965), Chap. 12.

${ }^{16}$ A.J. Legget, in Quantum Mechanics of Complex Systems I, Vol. 218 of NATO Advanced Study Institute, Series B: Physics, edited by D. Baeriswyl, A.R. Bishop, and J. Carmelo (Plenum New York, 1989); A.O. Caldeira and A.J. Legget, Physica A 121, 587 (1983).

${ }^{17}$ A. Schmid, J. Low Temp. Phys 49, 609 (1982).

${ }^{18}$ W. Brenig, H. Muller, and R. Sedlmeier, Phys. Lett. 54A, 109 (1975).

${ }^{19}$ P. Hedegård, Phys. Rev. B 35, 533 (1987).

${ }^{20}$ J. Rammer and H. Smith, Rev. Mod. Phys. 58, 2 (1986).

${ }^{21}$ Gerald D. Mahan, Many-Particle Physics, 2nd ed. (Plenum Press, New York, 1990).

${ }^{22}$ C.W. Gardiner, IBM J. Res. Dev. 32 1, (1988).

${ }^{23}$ A.I. Volokitin and B.N.J. Persson R.R. Cavanagh, E.J. Heilweil, and J.C. Stephenson, Surf. Sci. 299-300, 643 (1994).

${ }^{24}$ J. Roguzik, J. Kuppers, and V. Dose, Surf. Sci. 148, L653 (1984).

${ }^{25}$ G.D. Billing, Chem. Phys. 116, 269 (1987), and references therein. 